\title{
Insights into brain development and disease from neurogenetic analyses in Drosophila melanogaster
}

\author{
HEINRICH REICHERT \\ Biozentrum, University of Basel, Basel, Switzerland \\ (Email, heinrich.reichert@unibas.ch)
}

\begin{abstract}
Groundbreaking work by Obaid Siddiqi has contributed to the powerful genetic toolkit that is now available for studying the nervous system of Drosophila. Studies carried out in this powerful neurogenetic model system during the last decade now provide insight into the molecular mechanisms that operate in neural stem cells during normal brain development and during abnormal brain tumorigenesis. These studies also provide strong support for the notion that conserved molecular genetic programs act in brain development and disease in insects and mammals including humans.
\end{abstract}

[Reichert H 2014 Insights into brain development and disease from neurogenetic analyses in Drosophila melanogaster. J. Biosci. 39 595-603] DOI 10.1007/s12038-014-9444-x

\section{Introduction}

In his book entitled The Descent of Man Charles Darwin considered a central question posed by evolutionary theory, namely, how can it be, that the remarkable higher mental powers of man could have evolved from those of higher animals. From a series of extensive observations and considering the reports of numerous authorities, Darwin concluded that 'the difference in mind between man and the higher animals, great as it is, certainly is one of degree and not of kind' (Darwin 1871). This notion, that the differences in mind between man and animals is due to incremental and not fundamental differences, is borne out by the remarkable similarity in the basic neural anatomy of all vertebrate brains. The fact that brains from fish to mammals are composed of comparable anatomical substructures is widely accepted and indicates that all vertebrate brains derive evolutionarily from an ancestral brain in which these substructures were already present, albeit in a simplified form. In contrast, it is not clear if this similarity in brain structures also holds for vertebrates as compared to invertebrates such as insects. At the overt neuroanatomical level, the brains and central nervous systems of higher invertebrates, while highly complex, appear not to correspond anatomically to those of vertebrates.
However, when Santiago Ramon y Cajal examined the cell types and circuit features of the visual systems in insects and vertebrates, he noted striking similarities and concluded that 'the essential plan was maintained with small variations and retouches of adaptation' (Cajal and Sanchez 1915). Despite the fundamental importance of this observation and the resulting evolutionary implications, which suggest a monophyletic origin of all brains, experimental investigations into the validity of this notion were not possible at the beginning of the 20th century. In the 100 years since Cajal's groundbreaking observation, an important invertebrate genetic model system, namely Drosophila, has emerged and a vast array of genetic tools for analysing the development and function of the nervous system in this model system has been developed. To a significant degree, this was pioneered by a remarkable set of highly talented young scientists working with Seymour Benzer at Caltech, and among these was an Indian researcher who would play a prominent role in the development of Drosophila molecular biology and neurogenetics - Obaid Siddiqi. Siddiqi's work not only contributed to the powerful genetic toolkit that is now available for studying the nervous system of Drosophila experimentally, it also initiated a series of highly successful investigations into the development and function of the

Keywords. Asymmetric division; lineage; neuroblast; SWI/SNF complex; transit amplifying cell; tumour 
olfactory system of the fly carried out together with his student, Veronica Rodrigues.

With these new experimental tools and by taking advantage of the Drosophila neurogenetic model, it has been possible in recent years to reinvestigate Cajal's initial observation experimentally. Studies carried out during the last decade have provided insight into the mechanisms of brain development, and taken together, these insights provide strong support for the notion that insect and mammalian brain development is mediated by very similar and highly conserved genetic programs. In this review, I summarize the main steps in the development of the Drosophila brain and consider some of the similarities that brain development in the fly shares with brain development in mammals. I also review recent findings concerning aberrant developmental mechanisms notably those that occur in brain tumours, which indicate that similar pathological mechanisms operate in the brains of flies and mammals. Taken together, these findings indicate that brain development, be it normal or abnormal, is highly similar in insects and mammals and provides further support for evolutionarily conserved mechanisms of brain development and a common ancestral origin of all animal brains.

These developmental and evolutionary insights, as well as other equally important advances in areas such as neuroethology and behaviour, are due to the efforts of many excellent researchers over many decades. Obaid Siddiqi was a star among these excellent researchers, and the knowledge that he generated and passed on to future generations is perhaps the most appropriate tribute to the life and career of an exceptional scientist.
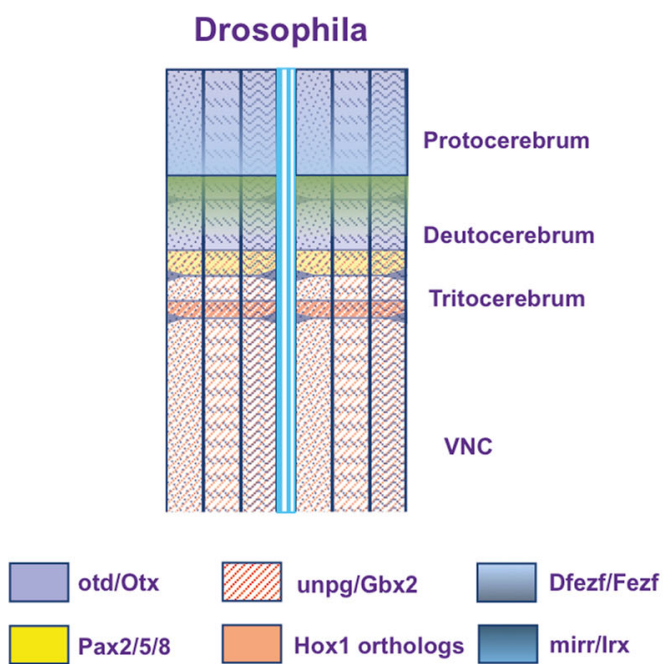

\section{Genesis: The neuroectoderm gives rise to neural stem cells}

The brain of Drosophila is a highly complex structure composed of tens of thousands of neurons, which are intricately interconnected in complex circuitry. All of these neurons derive from a restricted set of neural stem cell-like progenitors, called neuroblasts, which originate in the neuroectoderm of the early embryo (Urbach and Technau 2004; Doe 2008; Knoblich 2008; Egger et al. 2008; Reichert 2011; Homem and Knoblich 2012). The embryonic neuroectoderm is initially a sheet-like ensemble of epithelial cells. During embryonic development the cells of the neuroectoderm become regionalized in a spatially organized manner into domains of differential gene expression. This occurs through the action of developmental patterning genes that are expressed along the anterior-posterior and along the medial-lateral body axis. Their spatial overlap results in a Cartesian coordinate-like regionalization of the neuroectoderm such that different spatial domains of the neuroectoderm express different combinations of developmental control genes (Skeath and Thor 2003; Urbach and Technau 2004; Technau et al. 2006). In this manner positional information is imparted to the cells of the neuroectoderm in the form of a combinatorial gene expression code (figure 1).

Remarkably similar processes occur during regionalization of the neuroectoderm of vertebrates (Reichert and Simeone 2001; Lichtneckert and Reichert 2005, 2008; Reichert 2009). Homologous developmental control genes are involved, and the topological expression pattern of these

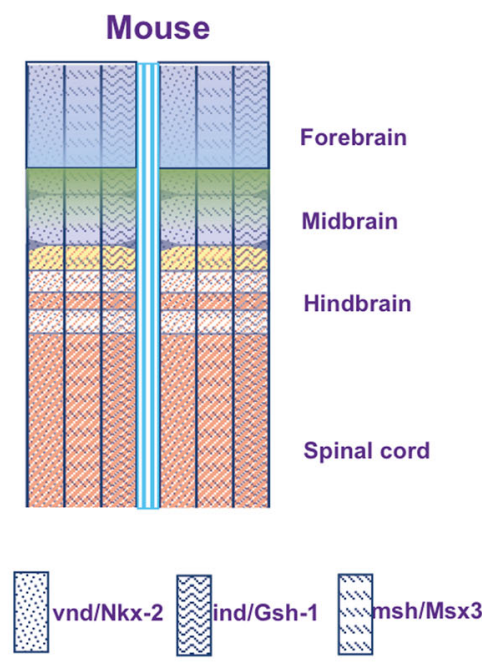

Figure 1. Regionalization of the neuroectoderm in Drosophila (left) and mouse (right). Developmental control genes, mainly transcription factors, are expressed along the anterior-posterior and medial-lateral (=dorsoventral) axis of the neuroectoderm, resulting in Cartesian coordinate-like domains with different combinations of genes. Homologous genes are expressed in similar topology in both the insect and the mammal neuroectoderm. Simplified summary scheme with gene expression domain colour-coded. Anterior-posterior genes: otd (Drosophila)/Otx (mouse), unpg/Gbx2, Dfezf/Fezf, Pax2/5/8, Hox1, mirr/Irx. Medial-lateral genes: vnd/Nkx-2, ind/Gsh-1, msh/Msx3. 
genes as well as the resulting combinatorial code in the vertebrate neuroectoderm are strikingly similar to those seen in the fly (figure 1). This conservation of genetic mechanisms for regionalization seen early in neurogenesis is underscored by cross-phylum genetic rescue experiments (Acampora et al. 1998, 2001; Leuzinger et al. 1998). In these experiments developmental control genes from the fly are transgenically expressed in mammalian embryos lacking the homologous vertebrate gene and can functionality replace the defect vertebrate homolog in these mutant animals. Thus, in terms of gene homology, gene expression pattern and gene function, there are remarkable similarities in the process of regionalization of the neuroectoderm in insects and mammals.

\section{Lineage: Neural stem cells proliferate to generate lineages of neurons}

Following the regionalization of the embryonic neuroectoderm in Drosophila, neural stem cell-like progenitors called neuroblasts derive from the neuroectoderm (Hartenstein and Wodarz 2013). These large primary progenitor cells are highly proliferative and typically undergo multiple rounds of asymmetric cell divisions, in which they self-renew and at the same time generate a smaller daughter cell called a GMC which divides once to produce two postmitotic neural cells (Doe 2008; Knoblich 2008; Egger et al. 2008; Reichert 2011). Due to its delamination from the neuroectoderm, each neuroblast largely retains the gene expression pattern of the neuroectodermal region from which it derives. In this manner each neuroblast acquires a specific combination of developmental control genes, usually comprising transcription factors, and hence, each of the approximately one hundred neuroblast pairs in the central brain of the fly manifests a specific combinatorial code determined by its position of origin in the neuroectoderm (Skeath and Thor 2003; Technau et al. 2006; Urbach and Technau 2004). This molecular code represents positional information and is involved in specification of each progenitor as well as of the neural cells that derive from the progenitor.

Through their proliferative activity, neuroblasts each generate a family-like lineage of neural progeny (figure 2). Thus, the central brain is a complex aggregation of approximately 100 pairs of neuroblast lineages (Yu et al. 2013; Ito et al. 2013; Wong et al. 2013). The neurons in each of these lineages generally manifest similar, albeit not identical, neuroanatomical features. For example, neurons of one lineage often show comparable dendritic innervation and axonal projection patterns. This is exemplified by the lineages, which generate the neurons that innervate the antenna lobe of the olfactory system (Rodrigues and Hummel 2008; Brochtrup and Hummel 2011; Das et al. 2011). Among the five neuroblast lineages that generate antennal lobe neurons, one lineage, termed ALv2, consists entirely of local interneurons that restrict all of their processes to the glomeruli of the antennal lobe. Similarly, a second antennal lobe lineage, ALad1, consists of projection interneurons, each of which
A

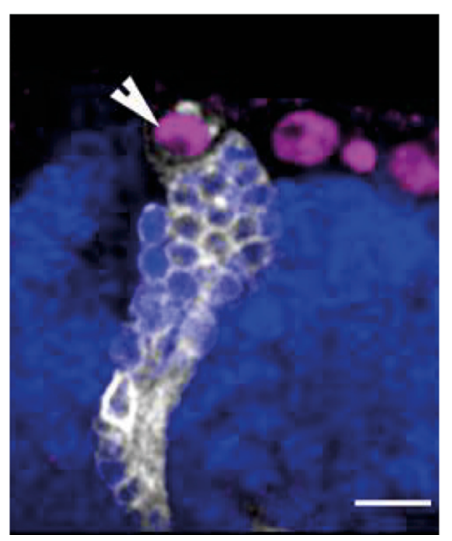

B

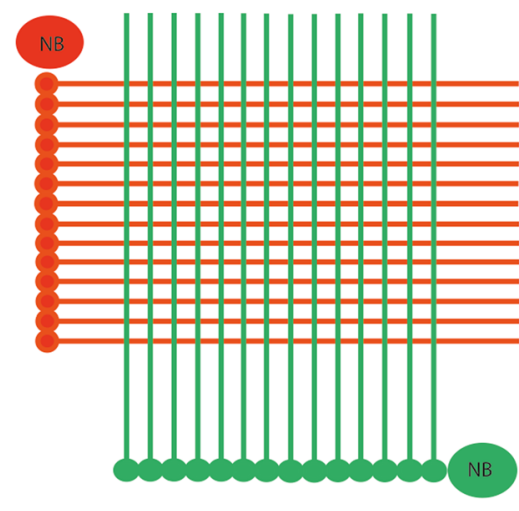

Figure 2. Proliferating neuroblasts generate lineages of neural progeny. (A) A neuroblast (arrowhead) generates a lineage of neural cells (outlined in white) in the larval brain of Drosophila. The parent neuroblast and its daughter cells are labelled using MARCM a clonal labelling technique (Lee and Luo 1999), and the neuroblast is co-immunolabelled with a neuroblast-specific marker. Neurons generate axons that project together. Scale bar, $10 \mu \mathrm{m}$ (courtesy B Bello). (B) Neuroblast lineages contribute to brain macrocircuitry. The lineage of neurons generated by a given neuroblast (NB) often project their axons to similar parts of the brain. Hence the neuroblast lineages form units of projection ('projection envelopes'). Simplified summary scheme of two neuroblasts, and their lineal daughter cells with axons. If the axon terminals of two neuroblast lineages project to the same target regions, synapses can form between the neurons of the two lineages. 
innervates a specific glomerulus of the antennal lobe and projects its axon to higher brain centers that include a center for olfactory learning called the mushroom body. The mushroom body in turn comprises four virtually identical neuroblast lineages, each of which generates similar sets of central complex intrinsic neurons (Ito and Hotta 1992; Lee and Luo 1999; Lee et al. 1999; Ito and Awasaki 2008). Interestingly, the neurons of the ALv2 lineage make synaptic connections with the neurons of the ALadl lineage, and the neurons of the ALad1 lineage make synaptic connections with the neurons of the mushroom body lineages (Wilson 2013). Thus, lineages not only represent units of neuroanatomical projection, they also represent the macrocircuitry of the brain (figure 2).

Positional information inherited by the neuroblast from the neuroectoderm and transmitted from the parent neuroblast to its progeny is important for specifying the identity of these lineally related neurons. In addition, two additional molecular processes operate in proliferating neuroblast lineages and are important for neuronal type diversity comprised in a given lineage. The first is represented by a generic temporal transcription factor series that operates in most neuroblasts. It consists of a serial cascade of transiently expressed transcription factors, each of which is expressed in the proliferating neuroblast during a specific time window (Maurange et al. 2008; Sousa-Nunes et al. 2010; Maurange 2012; Li et al. 2013). The GMC that is generated by the neuroblast during each time window inherits the expression of that transcription factor, and the neurons that derive from the GMC inherit and maintain the expression of the same transcription factor. This temporal information contributes to the specification of different cell types and hence results in birth-order-dependent neuronal cell type generation in each neuroblast lineage. The second generic mechanism operating in all neuroblast lineages involves the binary subdivision of a lineage into two molecularly different hemilineages. This is occurs through the asymmetric segregation of the Notchsignalling inhibitor Numb to one of the two neuronal daughters of each GMC division such that one daughter has active Notch signalling while the other daughter has inhibited Notch signalling (Karcavich and Doe 2005; Karcavich 2005). This difference translates into lineagespecific differences in the cellular and molecular properties of the two daughter cells and, in consequence, into two differentially fated hemilineages, one of which is Notch-On while the other is Notch-Off, in each neuroblast lineage (Kumar et al. 2009; Lin et al. 2010; Das et al. 2010; Truman et al. 2010). In this manner, binary Notch signalling together with temporal information and positional information provided by the neuroblast results in the specification of different cell fates in the lineages of the developing CNS.

\section{Complexity: Increasing neuronal number and diversity through transit amplifying cells}

Recent findings indicate that here are two different classes of neuroblasts in the Drosophila brain. Most neuroblasts belong to the type I class that generates their neuronal progeny directly through non-self-renewing GMCs (figure 3). In type I neuroblast lineages, one division of the parent neuroblast results, via one GMC, in the generation of two neural progeny. In contrast, neuroblasts of the type II class generate their neural progeny indirectly through intermediate neuronal progenitors (INPs), which function as transit amplifying cells (Bello et al. 2008; Bowman et al. 2008; Boone and Doe 2008; Weng and Lee 2011). Each INP can undergo a limited series of around five self-renewing divisions, each of which produces one GMC, which then divides once more to generate neuronal cells (figure 3). Hence, in type II neurobast lineages, one division of the parent neuroblasts results, via one INP and multiple GMCs, in approximately 10 neural progeny corresponding to a 5 -fold amplification of proliferation. Accordingly, type II neuroblast lineages comprise up to 500 neural cells, in contrast to type I lineages, which only contain 100-150 neural cells. This amplification of proliferation in type II lineages is especially important for generating the numerous neurons of a sensorimotor integration centre called the central complex, in which thousands of neurons are produced by only eight type II neuroblast pairs (Izergina et al. 2009; Jiang and Reichert 2012; Bayraktar et al. 2010; Yang et al. 2013; Riebli et al. 2013).

In addition to increased numbers of progeny, type II neuroblasts lineages also show a marked increase in diversity of neural cell types. For example, all type II neuroblasts generate both neurons and glial cell types and are, therefore, in functional terms multipotent neuroglioblasts (Viktorin et al. 2011, 2013). Moreover, the sub-lineage that each INP generates is composed of markedly different neural types (Wang et al. 2014). This diversification of cell types is controlled by an INPspecific temporal transcription factor series, which is functionally similar to the temporal series in neuroblasts but comprises a different transcription factor set (Bayraktar and Doe 2013). Since the same generic temporal transcription factor series operates in all the INPs of a type II neuroblast, each cell type is reproduced in each INP sublineage in a stereotyped birth-order-dependent manner. Thus, all first-born INP progeny are of a given type, all second born INP progeny are of a different type, and so forth. In this manner, type II lineages not only amplify neural cell number but also increase neural cell type diversity. Interestingly, two different types of primary progenitors are also found in the developing mammalian brain, and notably in the cerebral cortex (Brand and Livesey 2011; Fietz and Huttner 2011; Homem and 


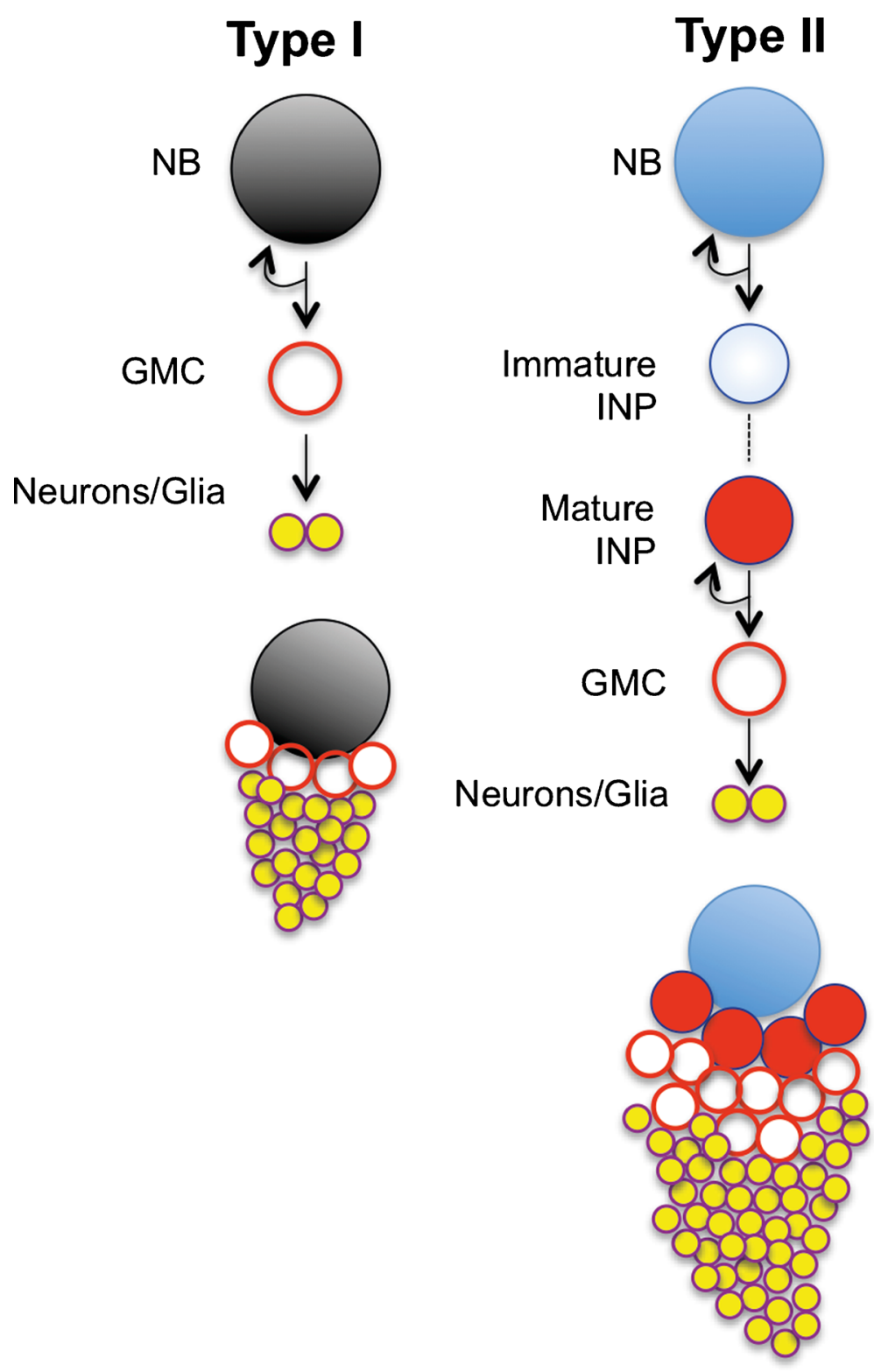

Figure 3. Two different types of neuroblast in the Drosophila brain, type I (left) and type II (right). Type I neuroblasts (NB) proliferate through non-self-renewing GMC daughter cells that divide once to produce two postmitotic neural cells (neurons or glial cells). Type I neuroblast lineages typically comprise 100-150 neural cells. Type II neuroblasts proliferate through transit amplifying cells, INPs, that undergo a maturation process and as mature INPs undergo limited number of proliferative divisions, each of which generates a GMC daughter that divides once. Type II neuroblast lineages comprise up to 500 neural cells (courtesy Kyung Hwa Kang).

Knoblich 2012). One type, corresponding to type I neuroblasts, generates its progeny primarily through nonself-renewing secondary progenitors that are comparable to GMCs and each give rise to two neural cells. The second type, OSVZ progenitors corresponding to type II neuroblasts, generates its progeny through self-renewing transit amplifying cells that are comparable to INPs and can undergo a limited series of proliferative divisions. This second type of primary progenitor is predominant in cortical development of higher mammals including primates, and the marked amplification of proliferation that these progenitors can achieve has been linked to evolution of the remarkably large, gyrencephalic neocortex of humans (Lui et al. 2011). 


\section{Aberration: Defects in neural stem cell proliferation cause brain tumours}

The unlimited proliferative potential of self-renewing neural stem cells must be tightly regulated in order to prevent uncontrolled overproliferation that can result in cancerous overgrowth. Indeed, many human tumours of the brain and other organs are thought to derive from misregulated primary or secondary progenitors, and this has given rise to the cancer stem cell concept of tumorigenesis (Reya et al. 2001; Kreso and Dick 2014). However, the molecular processes that are responsible for stem-cell-dependent tumour initiation in humans or mammalian models are poorly understood. In contrast, in Drosophila, a great deal is known about the molecular mechanisms underlying neural stem cell proliferation during normal development, and powerful genetic and genomic tools are available to investigate how the misregulation of these mechanisms might result in brain tumour formation.

In the last decade, a number of studies have shown that defects in the molecular control of asymmetric cell division of neuroblasts can result in uncontrolled proliferation and tumorigenesis (Caussinus and Gonzalez 2005; Januschke and Gonzalez 2008; Gonzalez 2013). Normal asymmetric cell division requires the correct operation of two molecular complexes in the dividing neuroblast (Gonzalez 2007;
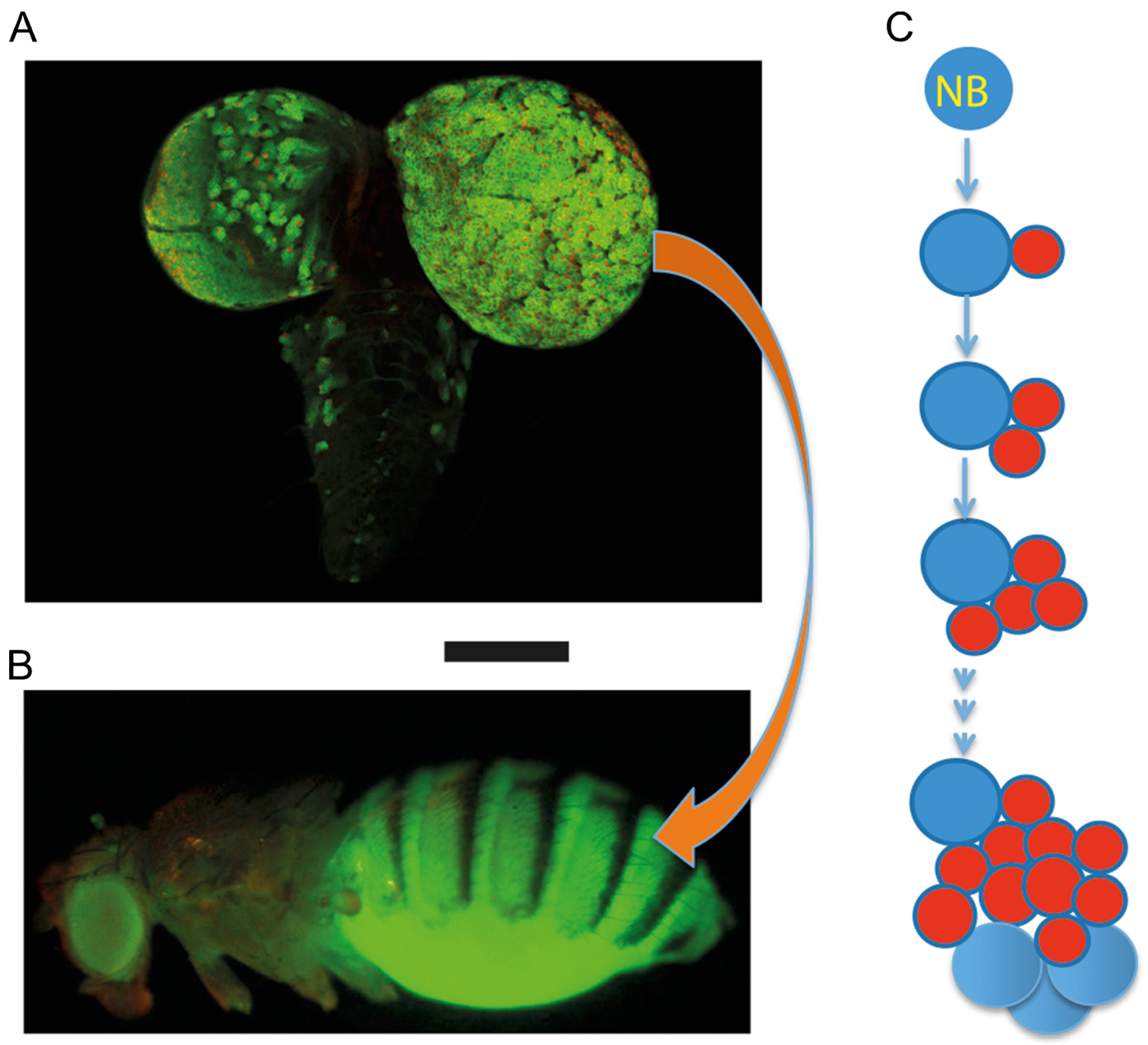

Figure 4. Tumorigenesis in SWI/SNF complex mutant type II neuroblast lineages. (A) A wild-type brain hemisphere (right) and a mutant brain hemisphere showing tumorigenic overproliferation (left); neuroblast lineages labelled with GFP (green fluorescent protein). Scale bar, $100 \mu \mathrm{m}$ (Courtesy of Yanrui Jiang). (B) Transplantation of GFP-labelled mutant brain tissue into the abdomen of a wild-type host fly results in a lethal large GFP-labeled tumour after several weeks. Scale bar, $500 \mu \mathrm{m}$. (C) In SWI/SNF complex mutant type II neuroblast lineages, type II neuroblasts (NB) generate defective INPs which accumulate and subsequently revert back to an ectopic neuroblast-like progenitor resulting in uncontrolled proliferation and tumorigenesis. 
Knoblich 2008, 2010). An apical complex is required for maintenance of polarity and spindle orientation as well as for segregation of cell fate determinants of a basal complex into the smaller daughter cell. These cell fate determinants, the transcription factor Prospero, the translational regulator Brat, and the Notch-signalling inhibitor Numb are required in the daughter cell to inhibit self-renewal and promote differentiation. Mutational inactivation of many of the molecules involved in asymmetric cell division, notably of the cell fate determinants, generally leads to uncontrolled overgrowth and transplantable tumours that acquire typical features of cancer such as immortality, resistance to apoptotic cell death, chromosome irregularity, and metastatic potential (Januschke and Gonzalez 2008; Gonzalez 2013; Neumüller and Knoblich 2009; Knoblich 2010).

Recently, further insight into the molecular mechanisms of brain tumour formation has been obtained in Drosophila by combining genome-wide RNAi screens for genes involved in neuroblast overproliferation with methods for isolation and purification of normal and abnormal neuroblasts (Neumüller et al. 2011; Berger et al. 2012). These studies show that the type II neuroblasts, which amplify proliferation through INPs, are especially vulnerable to tumorigenic misregulation (figure 4). Moreover, they identify a master chromatin remodelling machinery, the SWI/SNF complex, as a major element in the control of proliferation in the type II lineages (Eroglu et al. 2014). During normal brain development, the SWI/SNF complex promotes correct developmental directionality in these lineages and prevents the aberrant reversion of INPs back to neuroblast-like cells with unlimited self-renewal activity. This is achieved, at least in part, through activation of a self-renewal restriction program in the INP during the maturation processes, which is required before the INP can begin its transient proliferation phase (figure 4). Mutations in any of the SWI/SNF complex genes disrupt this critical control of lineage directionality and selfrenewal restriction, and in consequence, INPs revert to an uncontrolled proliferating ectopic progenitor type that results in tumorigenesis (Eroglu et al. 2014). In view of the remarkable conservation of developmental mechanisms in flies and vertebrates including man, it is perhaps not surprising that mutations in the SWI/SNF chromatin remodelling complex are among the most frequently found mutations in many types of human cancers. From this perspective, the analysis of the mechanisms by which SWI/SNF complex genes act in suppressing tumorigenesis in Drosophila neural stem cell lineages may help in analysing their corresponding molecular roles in human cancer biology.

\section{Conclusion}

The remarkable advances in our understanding of the Drosophila brain, coupled with equally remarkable advances in the genetic, genomic and transgenic methods available for studying Drosophila, would not have been possible without the pioneering work of many gifted and dedicated scientists like Obaid Siddiqi. Thanks to their work, we now have an initial understanding of mechanisms responsible for normal and abnormal brain development in this neurogenetic model. The findings obtained in the Drosophila model during the last decade fully support the observation made by Cajal 100 years ago, in that the differences between developmental mechanisms that operate in the brains of flies and mammals, in health and in disease, are indeed 'small variations and retouches of adaptations', variations on common, evolutionarily conserved themes. In this sense, they also confirm Darwin's notion that these differences, large or small, are 'one of degree and not of kind'.

\section{Acknowledgements}

The author acknowledges the support of the Swiss NSF.

\section{References}

Acampora D, Avantaggiato V, Tuorto F, Barone P, Reichert H, Finkelstein R and Simeone A 1998 Murine Otx1 and Drosophila otd genes share conserved genetic functions required in invertebrate and vertebrate brain development. Dev. $1251691-$ 1702

Acampora D, Boyl PP, Signore M, Martinez-Barbera JP, Ilengo C, Puelles E, Annino A, Reichert H, Corte $\mathrm{G}$ and Simeone A 2001 OTD/OTX2 functional equivalence depends on $5^{\prime}$ and 3' UTR-mediated control of Otx2 mRNA for nucleo-cytoplasmic export and epiblast-restricted translation. Dev. 128 4801-4813

Bayraktar OA, Boone JQ, Drummond ML and Doe CQ 2010 Drosophila type II neuroblast lineages keep Prospero levels low to generate large clones that contribute to the adult brain central complex. Neural. Dev. 526

Bayraktar OA and Doe CQ 2013 Combinatorial temporal patterning in progenitors expands neural diversity. Nature $498449-455$

Bello BC, Izergina N, Caussinus E and Reichert H 2008 Amplification of neural stem cell proliferation by intermediate progenitor cells in Drosophila brain development. Neural. Dev. 35

Berger C, Harzer H, Burkard TR, Steinmann J, van der Horst S, Laurenson AS, Novatchkova M, Reichert H, et al. 2012 FACS purification and transcriptome analysis of Drosophila neural stem cells reveals a role for Klumpfuss in self-renewal. Cell Rep. 2 407-418

Boone JQ and Doe CQ 2008 Identification of Drosophila type II neuroblast lineages containing transit amplifying ganglion mother cells. Dev. Neurobiol. 68 1185-1195

Bowman SK, Rolland V, Betschinger J, Kinsey KA, Emery G and Knoblich JA 2008 The tumor suppressors Brat and Numb regulate transit-amplifying neuroblast lineages in Drosophila. Dev. Cell 14 535-546 
Brand AH and Livesey FJ 2011 Neural stem cell biology in vertebrates and invertebrates: more alike than different? Neuron $\mathbf{7 0}$ 719-729

Brochtrup A and Hummel T 2011 Olfactory map formation in the Drosophila brain: genetic specificity and neuronal variability. Curr. Opin. Neurobiol. 21 85-92

Cajal S and Sanchez D 1915 Contribución al conocimiento de los centros nerviosos de los insectos. Trab. Lab. Inv. Biol. 13 1-68

Caussinus E and Gonzalez C 2005 Induction of tumor growth by altered stem-cell asymmetric division in Drosophila melanogaster. Nat. Genet. 37 1125-1129

Darwin C 1871 The descent of man, and selection in relation to sex (John Murray: London)

Das A, Chiang A, Davla S, Priya R, Reichert H, Vijayraghavan K and Rodrigues V 2011 Identification and analysis of a glutamatergic local interneuron lineage in the adult Drosophila olfactory system. Neural. Syst. Circuits 14

Das A, Reichert H and Rodrigues V 2010 Notch regulates the generation of diverse cell types from the lateral lineage of Drosophila antennal lobe. J. Neurogenet. 24 42-53

Doe CQ 2008 Neural stem cells: balancing self-renewal with differentiation. Dev. 135 1575-1587

Egger B, Chell JM and Brand A 2008 Insights into neural stem cell biology from flies. Philos. Trans. R. Soc. Lond. B. Biol. Sci. 363 $39-56$

Eroglu E, Burkhard TR, Jiang Y, Saini N, Homem CCF, Reichert H and Knoblich JA 2014 SWI/SNF complex prevents lineage reversion and induces temporal patterning in neural stem cells. Cell 156 1259-1273

Fietz SA and Huttner WB 2011 Cortical progenitor expansion, selfrenewal and neurogenesis-a polarized perspective. Curr. Opin. Neurobiol. 21 23-35

Gonzalez C 2007 Spindle orientation, asymmetric division and tumour suppression in Drosophila stem cells. Nat. Rev. Genet. $8462-472$

Gonzalez C 2013 Drosophila melanogaster: a model and a tool to investigate malignancy and identify new therapeutics. Nat. Rev. Cancer. 13 172-183

Hartenstein V and Wodarz A 2013 Initial neurogenesis in Drosophila. Wiley. Interdiscip. Rev. Dev. Biol. 2 701-721

Homem CC and Knoblich JA 2012 Drosophila neuroblasts: a model for stem cell biology. Dev. 139 4297-4310

Ito K and Hotta Y 1992 Proliferation pattern of postembryonic neuroblasts in the brain of Drosophila melanogaster. Dev. Biol. 149 134-148

Ito $\mathrm{K}$ and Awasaki T 2008 Clonal unit architecture of the adult fly brain. Adv. Exp. Med. Biol. 628 137-158

Ito M, Masuda N, Shinomiya K, Endo K and Ito K 2013 Systematic analysis of neural projections reveals clonal composition of the Drosophila brain. Curr. Biol. 23 644-655

Izergina N, Balmer J, Bello B and Reichert H 2009 Postembryonic development of transit amplifying neuroblast lineages in the Drosophila brain. Neural Dev. 444

Januschke J and Gonzalez C 2008 Drosophila asymmetric division, polarity and cancer. Oncogene 27 6994-7002

Jiang Y and Reichert H 2012 Programmed cell death in type II neuroblast lineages is required for central complex development in the Drosophila brain. Neural Dev. 73
Karcavich RE 2005 Generating neuronal diversity in the Drosophila central nervous system: a view from the ganglion mother cells. Dev. Dyn. 232 609-616

Karcavich R and Doe CQ 2005 Drosophila neuroblast 7-3 cell lineage: a model system for studying programmed cell death, Notch/Numb signaling, and sequential specification of ganglion mother cell identity. J. Comp. Neurol. 481 240-251

Knoblich JA 2008 Mechanisms of asymmetric stem cell division. Cell 132 583-597

Knoblich JA 2010 Asymmetric cell division: recent developments and their implications for tumour biology. Nat. Rev. Mol. Cell. Biol. 11 849-860

Kreso A and Dick JE 2014 Evolution of the Cancer Stem Cell Model. Cell Stem Cell 14 275-291.

Kumar A, Bello B and Reichert H 2009 Lineage-specific cell death in postembryonic brain development of Drosophila. Dev. 136 3433-3442

Lee T, Lee A and Luo L 1999 Development of the Drosophila mushroom bodies: sequential generation of three distinct types of neurons from a neuroblast. Dev. 126 4065-4076

Lee T and Luo L 1999 Mosaic analysis with a repressible cell marker for studies of gene function in neuronal morphogenesis. Neuron 22 451-461

Leuzinger S, Hirth F, Gerlich D, Acampora D, Simeone A, Gehring WJ, Finkelstein R, Furukubo-Tokunaga K, et al. 1998 Equivalence of the fly orthodenticle gene and the human OTX genes in embryonic brain development of Drosophila. Dev. 125 17031710

Li X, Chen Z and Desplan C 2013 Temporal patterning of neural progenitors in Drosophila. Curr. Top. Dev. Biol. 105 69-96

Lichtneckert R and Reichert H 2005 Insights into the urbilaterian brain: conserved genetic patterning mechanisms in insect and vertebrate brain development. Heredity 94 465-477

Lichtneckert R and Reichert H 2008 Anteroposterior regionalization of the brain: genetic and comparative aspects. Adv. Exp. Med. Biol. 628 32-41

Lin S, Lai SL, Yu HH, Chihara T, Luo L and Lee T 2010 Lineagespecific effects of Notch/Numb signaling in post-embryonic development of the Drosophila brain. Dev. 137 43-51

Lui JH, Hansen DV and Kriegstein AR 2011 Development and evolution of the human neocortex. Cell 146 18-36

Maurange C 2012 Temporl specification of neural stem cells: insights from Drosophila neuroblasts. Curr. Top. Dev. Biol. 98 199-228

Maurange, Cheng L and Gould AP 2008 Temporal transcription factors and their targets schedule the end of neural proliferation in Drosophila. Cell 133 891-902

Neumüller RA and Knoblich JA 2009 Dividing cellular asymmetry: asymmetric cell division and its implications for stem cells and cancer. Genes Dev. 23 2675-2699

Neumüller RA, Richter C, Fischer A, Novatchkova M, Neumüller KG and Knoblich JA 2011 Genome-wide analysis of selfrenewal in Drosophila neural stem cells by transgenic RNAi. Cell Stem Cell 8 580-593

Reichert H 2009 Evolutionary conservation of mechanisms for neural regionalization, proliferation and interconnection in brain development. Biol. Lett. 5 112-116 
Reichert H 2011 Drosophila neural stem cells: cell cycle control of self-renewal, differentiation, and termination in brain development. Results Probl. Cell. Differ. 53 529-546

Reichert H and Simeone A 2001 Developmental genetic evidence for a monophyletic origin of the bilaterian brain. Philos. Trans. R. Soc. Lond. B Biol. Sci. 356 1533-1544

Reya T, Morrison SJ, Clarke MF and Weissman I 2001 Stem cells, cancer, and cancer stem cells. Nature 414 105-111

Riebli N, Viktorin G and Reichert H 2013 Early-born neurons in type II neuroblast lineages establish a larval primordium and integrate into adult circuitry during central complex development in Drosophila. Neural Dev. 86

Rodrigues V and Hummel T 2008 Development of the Drosophila olfactory system. Adv. Exp. Med. Biol. 628 82-101

Skeath JB and Thor S 2003 Genetic control of Drosophila nerve cord development. Curr. Opin. Neurobiol. 13 8-15

Sousa-Nunes R, Cheng LY and Gould AP 2010 Regulating neural proliferation in the Drosophila CNS. Curr. Opin. Neurobiol. 20 $50-57$

Technau GM, Berger C and Urbach R 2006 Generation of cell diversity and segmental pattern in the embryonic central nervous system of Drosophila. Dev. Dyn. 235 861-869

Truman JW, Moats W, Altman J, Marin EC and Williams DW 2010 Role of Notch signaling in establishing the hemilineages of secondary neurons in Drosophila melanogaster. Dev. 137 $53-61$

Urbach R and Technau GM 2004 Neuroblast formation and patterning during early brain development in Drosophila. Bioessays 26 739-751
Viktorin G, Riebli N, Popkova A, Giangrande A and Reichert H 2011 Multipotent neural stem cells generate glial cells of the central complex through transit amplifying intermediate progenitors in Drosophila brain development. Dev. Biol. 356 553-565

Viktorin, Riebli N and Reichert H 2013 A multipotent transitamplifying neuroblast lineage in the central brain gives rise to optic lobe glial cells in Drosophila. Dev. Biol. 379182 194

Wang YC, Yang JS, Johnston R, Ren Q, Lee YJ, Luan H, Brody T, Odenwald WF and Lee T 2014 Drosophila intermediate neural progenitors produce lineage-dependent related series of diverse neurons. Dev. 141 253-258

Weng M and Lee CY 2011 Keeping neural progenitor cells on a short leash during Drosophila neurogenesis. Curr. Opin. Neurobiol. 21 36-42

Wilson RI 2013 Early olfactory processing in Drosophila: mechanisms and principles. Annu. Rev. Neurosci. 36 217-241

Wong DC, Lovick JK, Ngo KT, Borisuthirattana W, Omoto JJ and Hartenstein V 2013 Postembryonic lineages of the Drosophila brain: II. Identification of lineage projection patterns based on MARCM clones. Dev. Biol. 384 258-289

Yang JS, Awasaki T, Yu HH, He Y, Ding P, Kao JC and Lee T 2013 Diverse neuronal lineages make stereotyped contributions to the Drosophila locomotor control center, the central complex. J. Comp. Neurol. 521 2645-2662

Yu HH, Awasaki T, Schroeder MD, Long F, Yang JS, He Y, Ding $\mathrm{P}$, et al. 2013 Clonal development and organization of the adult Drosophila central brain. Curr. Biol. 23 633-643 